\title{
Bodily pain, social support, depression symptoms and stroke history are independently associated with sleep disturbance among the elderly: a cross-sectional analysis of the Fujiwara-kyo study
}

\author{
Yuko Kishimoto $^{1} \cdot$ Nozomi Okamoto $^{1} \cdot$ Keigo Saeki $^{1} \cdot$ Kimiko Tomioka $^{1} \cdot$ \\ Kenji Obayashi $^{1} \cdot$ Masayo Komatsu $^{1} \cdot$ Norio Kurumatani $^{1}$
}

Received: 11 November 2015/ Accepted: 29 March 2016/Published online: 12 April 2016

(C) The Japanese Society for Hygiene 2016

\begin{abstract}
Objective To investigate independent effects of various factors associated with sleep disturbance among community-dwelling elderly individuals.

Methods We analyzed data obtained from 3732 individuals aged $\geq 65$ years who responded to a self-administered questionnaire and participated in a structured interview which assessed the Pittsburgh Sleep Quality Index (PSQI), subjective bodily pain, the Jichi Medical School Social Support Scale, the Geriatric Depression Scale (GDS-15), health status, and demographic characteristics. Sleep disturbance was defined as a global PSQI score $>5.5$, which was used as a dependent variable in multiple logistic regression analysis to determine adjusted odds ratios (ORs) and $95 \%$ confidence intervals (CIs) of related factors.

Results We identified a significant increase in the adjusted ORs for female (OR 1.56, $95 \%$ CI 1.34-1.83), age $\geq 80$ years $(1.31,1.01-1.69)$, history of stroke (1.44, $1.08-1.92$ ), and a GDS- 15 score $\geq 6$ as compared to $0-2$ $(2.29,1.86-2.81)$, with regard to sleep disturbance. Participants with severe or very severe bodily pain had the highest adjusted OR $(3.00,2.15-4.19)$, and those with very mild bodily pain also had a relatively high OR (1.30, $1.06-1.60$ ), relative to those without subjective bodily pain. In addition, compared with participants with strong social support from spouse or family, those with weak social support had significantly increased adjusted ORs (1.21, $1.01-1.44,1.44,1.23-1.70$, respectively).
\end{abstract}

Norio Kurumatani

knorio@naramed-u.ac.jp

1 Department of Community Health and Epidemiology, Nara Medical University School of Medicine, 840 Shijo-cho, Kashihara, Nara 634-8521, Japan
Conclusions The present study indicates that sleeping disturbances among the elderly are closely associated with social support from a spouse and family. They are also associated with pain, even at stages in which subjective bodily pain is very mild.

Keywords Bodily pain · Elderly people $\cdot$ PSQI $\cdot$ Sleep disturbance $\cdot$ Social support

\section{Introduction}

Among community-dwelling elderly people aged 65 years and older, roughly one-fourth to one-third complain of some form of sleep disturbance $[1,2]$. According to a metaanalysis [3], both total sleep time and sleep efficiency decrease with age, whereas awake time and wake after sleep onset increase. Sleep disturbance affects physical health, and has also been shown to correlate with mental health [4].

Many epidemiological studies have found a variety of factors to be associated with sleep disturbances, including chronic conditions such as diabetes [5], hypertension [6], stroke [7], myocardial infarction [6], chronic kidney disease [8], depression [9], cognitive impairments [10], pain [11], lifestyle habits such as drinking [12], smoking [13], and certain physical activities (PAs) [14]. However, these studies have mostly focused on youth and adult populations $[6,8,12,13,15]$. Reports on elderly individuals are scarce, and tend to focus only on a limited number of related factors $[5,13]$. Old age is characterized by a high prevalence of chronic diseases. In addition, after retirement from their professional roles, elderly individuals tend to experience a reduction in the size of social networks [16], a decrease in interpersonal ties [16], and consequently, 
diminished social support. Diminished social support can affect one's state of mind and contribute to sleep disturbances [17].

In the context described above, the present study aimed to determine independent effects of various factors (including these characteristics of old age) associated with sleep disturbance among community-dwelling elderly individuals. To assess sleep disturbance, more than a few studies have used original questionnaires consisting of several items regarding whether there are insomnia symptoms or not $[2,18]$. In the present study, we used the Pittsburgh Sleep Quality Index (PSQI), which is a standardized questionnaire widely used in sleep-related epidemiological studies [19]. The validity of its Japanese version has been confirmed previously [9].

\section{Methods}

\section{Subjects}

Subjects of this study were participants of the Fujiwara-kyo study, a prospective cohort study on successful aging in Japanese community-dwelling people aged $\geq 65$ years [20]. The entry criteria included participants living in their own homes, were able to walk without assistance, and provided written informed consent. Potential participants were recruited with the cooperation of local resident associations and elderly clubs in the cities of Nara, Kashihara, YamatoKoriyama, and Kashiba in the northwestern part of Nara prefecture. Nara prefecture is where the first capital of Japan, called Fujiwara-kyo, was established. A total of 4427 (2174 men and 2253 women) participants completed a self-administered questionnaire survey and were interviewed by trained staff members before undergoing blood tests and blood pressure, height, and weight measurements. All participants underwent baseline examinations between June 2007 and October 2008.

This study was approved by the ethics committee of Nara Medical University, and written informed consent was obtained from all participants.

\section{Self-reported measure of sleep quality}

We used the PSQI self-reported questionnaire to evaluate participants' subjective sleep quality over the previous month. The PSQI consists of 19 self-rated questions which are grouped into 7 dimensions: subjective sleep quality, sleep latency, sleep duration, habitual sleep efficiency, sleep disturbances, use of sleeping medications, and daytime dysfunction. Each of the seven dimensions is rated on a scale of $0-3$, and then all ratings are summed to yield a global PSQI score, which ranges from 0 to 21 ; the higher the global score, the worse the sleep quality. This global score has been used in many epidemiological studies [21, 22]. The Japanese version [23] of the PSQI has been confirmed for its reliability and validity among people clinically diagnosed with sleep disturbance, as defined by a global score cutoff of 5.5. The sensitivity and specificity of the Japanese version of the PSQI have been reported to be 85.7 and $86.6 \%$, respectively [9], similar to those obtained in other countries [24].

\section{Other self-administered questionnaire surveys and interview}

We mailed to each participant a booklet containing the self-administered questionnaire over 50 pages long, prior to baseline interviews performed by trained medical staff. The questionnaire items covered many aspects of health conditions, lifestyle habits, and social support, in addition to demographic characteristics such as sex and age.

Participants were asked about past and present histories of five commonly occurring diseases among the elderly: stroke with clinical symptoms, acute myocardial infarction (AMI), cancer of any kind, hypertension, and diabetes. The degree of subjective pain, as described in the MOS 36-item short-form health survey (SF-36), was assessed on a sixpoint scale (none, very mild, mild, moderate, severe, and very severe) in response to the following question: "How much bodily pain have you had during the past 4 weeks?" [25]. The question was not created as part of the pain scale and has not been not validated, but the answer options were presented as graded scales which are commonly used for assessing pain [26]. The Japanese version of the SF36 has been established [27]. Depressive symptoms were evaluated using a shorter, 15-item version of the Geriatric Depression Scale (GDS-15) [28]. In previous validation studies, a score of $\geq 6$ suggested probable clinical depression in older Japanese individuals [29]. Cognitive function was examined using the mini-mental state examination (MMSE), with a score of $<24$ defined as cognitive impairment [30, 31]. Social support was evaluated using the Jichi Medical School Social Support Scale (JMS-SSS) [32], a 28-item questionnaire (8 items for support from spouse and 10 items each for support from family and friends) developed for measuring the availability of social support for community residents.

Information regarding self-reported PAs was obtained through the Japanese version of the International Physical Activity Questionnaire (the usual 7-day, short, self-administered version). According to the official IPAQ guidelines [33], PAs were estimated using responses to three different intensities of activities (i.e., vigorous intensity, moderate intensity, and walking) and the total amount of time spent engaged in each type of activity per 
week. Total weekly PAs (MET-min/week) was estimated by adding the products of reported time for each item by a MET value that is specific to each category of PA with some modifications for elderly people [34]. Average daily amount of ethanol intake (g/day) was estimated according to the type, frequency, and amount of alcohol participants consumed per week in the past 6 months. Smoking status was categorized as non-smoker, ex-smoker, and current smoker.

\section{Blood tests}

Venous blood samples were collected after overnight fasting. Glycated hemoglobin (HbA1c: National Glycohemoglobin Standardization Program value), plasma glucose, and serum creatinine were measured at a commercial laboratory (SRL Co. Inc., Tokyo, Japan) using a standard clinical chemistry analyzer. In this study, diabetes was defined as physician-diagnosed diabetes with medical treatment, or an HbA1c of $6.5 \%$ or higher at baseline without physician-diagnosed diabetes. The estimated glomerular filtration rate (eGFR) was calculated with serum creatinine using a formula from the Japanese Society of Nephrology-Chronic Kidney Disease Practice Guide [35]. Renal dysfunction was defined as eGFR $<60 \mathrm{ml} / \mathrm{min} /$ $1.73 \mathrm{~m}^{2}$.

\section{Statistical analysis}

Multiple comparisons of median global scores across 5-year age groups were performed using the KruskalWallis test because of skewed data. Means and medians between PSQI $>5.5$ (sleep disturbance) and PSQI $\leq 5.5$ groups were compared using the unpaired $t$ test and MannWhitney $U$ test, respectively. The chi square test was used for comparisons of categorical data.

Factors associated with sleep disturbance were examined using logistic regression analysis. Sleep disturbance status was set as a dependent variable, with independent variables listed in Table 2. As linearity with sleep disturbance status was not assured, continuous variables were categorized according to age $(65-69,70-74,75-79, \geq 80)$, MET value (MET-min/week: $<60,<300, \geq 300$ ), alcohol consumption (g/day: $0, \geq 0.01, \geq 20, \geq 40$ ) and eGFR (ml/ $\left.\min / 1.73 \mathrm{~m}^{2}:<60, \geq 60,<90, \geq 90\right)$. Participants with a GDS score $<6$ were subdivided into normal (0-2) and slightly depressive (3-5) [36]. With regard to the JMS-SSS, participants were categorized into two groups (weak and strong) based on the mean score of each of the three subscales (spouse, family, and friends) as a cutoff point. First, in a sex- and 5-year age-adjusted logistic regression model, independent variables associated with sleep disturbance were evaluated using prevalence odds ratio (OR), also employing the $95 \%$ confidence interval $(\mathrm{CI})$ and $P$ value. Second, all independent variables with a $P$ value $\leq 0.2$ in the first step were included in the multiple logistic model and mutually adjusted using the forced entry method. The Hosmer-Lemeshow statistic was used for examining fitness of the model to the actual data.

All statistical analyses were performed using SPSS version 21.0 for Windows (IBM SPSS Inc., IL, USA). A two-sided $P$ value $<0.05$ was considered statistically significant.

\section{Results}

A total of 3732 participants (1882 men and 1850 women) were subject to analysis, after excluding 153 whose responses to the questionnaire were incomplete, 298 with undetermined global PSQI scores due to missing data, 155 who did not provide any index of subjective pain on the SF36, and 89 who omitted answers to other necessary questions for the logistic regression model.

Table 1 shows summary statistics of global PSQI scores by sex and 5-year age groups. In both sexes, higher age groups had a higher mean score, with women scoring higher than men. Sleep disturbance was prevalent in $30.8 \%$ (579) of men and $41.5 \%$ (767) of women, with a global PSQI score of $>5.5$. In men, the prevalence of sleep disturbance increased from 28.1 to $36.3 \%$ with increasing age. In women, the prevalence also increased from 37.9 to $50.3 \%$ with increasing age; the values were much higher in women than in men in all 5-year age groups.

Table 2 compares selected characteristics of potential sleep-affecting factors between two groups according to

Table 1 Summary statistics of global PSQI (Pittsburgh Sleep Quality Index) scores by sex and 5-year age groups among 3732 participants aged $\geq 65$ years

\begin{tabular}{llrlll}
\hline \multicolumn{1}{l}{ Age } & No. & Mean & SD & Prevalence $(\%)^{\mathrm{a}}$ \\
\hline Men & & & & & \\
& $65-69$ & 612 & 4.37 & 2.62 & 28.1 \\
& $70-74$ & 670 & 4.62 & 2.83 & 30.9 \\
& $75-79$ & 388 & 4.78 & 2.95 & 31.7 \\
& $80 \leq$ & 212 & 5.04 & 3.09 & 36.3 \\
& Subtotal & 1882 & 4.62 & 2.83 & 30.8 \\
Women & & & & & \\
& $65-69$ & 670 & 5.26 & 3.16 & 37.9 \\
& $70-74$ & 606 & 5.49 & 3.17 & 41.4 \\
& $75-79$ & 385 & 5.65 & 3.44 & 43.4 \\
& $80 \leq$ & 189 & 5.82 & 3.24 & 50.3 \\
& Subtotal & 1850 & 5.47 & 3.23 & 41.5 \\
\hline
\end{tabular}

Scores range from 0 to 21

a Global PSQI score > 5.5 
Table 2 Comparisons of selected characteristics of potential sleep-affecting factors between two groups according to global PSQI score

\begin{tabular}{|c|c|c|c|c|c|}
\hline & \multicolumn{2}{|c|}{ PSQI $\leq 5.5(n=2386)$} & \multicolumn{2}{|c|}{ PSQI $>5.5(n=1346)$} & \multirow[t]{2}{*}{$P$ value } \\
\hline & No. (mean) & $\%(\mathrm{SD})$ & No. (mean) & $\%(\mathrm{SD})$ & \\
\hline Women & 1083 & 45.4 & 767 & 57.0 & 0.00 \\
\hline Age & $(72.2)$ & $(5.2)$ & $(72.7)$ & $(5.4)$ & $0.00^{\mathrm{b}}$ \\
\hline \multicolumn{6}{|l|}{ Current health condition } \\
\hline \multicolumn{6}{|l|}{ Depression } \\
\hline $\mathrm{GDS}^{\mathrm{c}} \geq 6$ & 256 & 10.7 & 326 & 24.2 & 0.00 \\
\hline \multicolumn{6}{|l|}{ Cognitive impairment } \\
\hline $\operatorname{MMSE}^{\mathrm{d}}<24$ & 104 & 4.4 & 83 & 6.2 & 0.02 \\
\hline \multicolumn{6}{|l|}{ Renal dysfunction } \\
\hline $\mathrm{eGFR}^{\mathrm{e}}<60$ & 574 & 24.1 & 381 & 28.3 & 0.02 \\
\hline \multicolumn{6}{|l|}{ Subjective bodily pain ${ }^{f}$} \\
\hline Moderate or more severe & 326 & 13.6 & 363 & 26.9 & 0.00 \\
\hline \multicolumn{6}{|l|}{ History of chronic conditions } \\
\hline \multicolumn{6}{|l|}{ Stroke } \\
\hline Presence history & 117 & 4.9 & 101 & 7.5 & 0.00 \\
\hline \multicolumn{6}{|l|}{ Myocardial infarction } \\
\hline Presence history & 53 & 2.2 & 38 & 2.8 & 0.25 \\
\hline \multicolumn{6}{|l|}{ Diabetes } \\
\hline $\mathrm{Yes}^{\mathrm{g}}$ & 343 & 14.4 & 200 & 14.9 & 0.69 \\
\hline \multicolumn{6}{|l|}{ Hypertension } \\
\hline Yes $^{\mathrm{h}}$ & 1656 & 69.4 & 929 & 69.0 & 0.81 \\
\hline \multicolumn{6}{|l|}{ Cancer of any kind } \\
\hline Presence history & 218 & 9.1 & 138 & 10.3 & 0.27 \\
\hline \multicolumn{6}{|l|}{ Social support } \\
\hline \multicolumn{6}{|l|}{ Spouse } \\
\hline Weak & 487 & 20.4 & 391 & 29.0 & 0.00 \\
\hline \multicolumn{6}{|l|}{ Family } \\
\hline Weak & 565 & 23.7 & 431 & 32.0 & 0.00 \\
\hline \multicolumn{6}{|l|}{ Friends } \\
\hline Weak & 550 & 23.1 & 388 & 28.8 & 0.00 \\
\hline \multicolumn{6}{|l|}{ Lifestyle habits } \\
\hline \multicolumn{6}{|l|}{ Physical activity } \\
\hline METs-min/week ${ }^{\mathrm{i}}$ & $(242.8)$ & $(234.2)$ & $(216.5)$ & (202.4) & $0.00^{\mathrm{b}}$ \\
\hline \multicolumn{6}{|l|}{ Alcohol } \\
\hline Current drinker & 958 & 40.2 & 466 & 34.6 & 0.00 \\
\hline Ethanol consumption (g/day) & $(12.4)$ & $(21.7)$ & $(9.5)$ & $(18.1)$ & $0.00^{\mathrm{b}}$ \\
\hline \multicolumn{6}{|l|}{ Smoking } \\
\hline Current smoker & 254 & 10.7 & 103 & 7.7 & 0.00 \\
\hline
\end{tabular}

a $\chi^{2}$ test

b $t$ test

c Geriatric Depression Scale (GDS-15)

d Mini-mental state examination

e Estimated glomerular filtration rate $\left(\mathrm{ml} / \mathrm{min} / 1.73 \mathrm{~m}^{2}\right)$

${ }^{\mathrm{f}}$ Bodily pain in the MOS 36-item short-form health survey

g Physician-diagnosed diabetes with medical treatment or HbA1c $>6.5 \%$ at baseline

h Currently treated for SBP $>140 \mathrm{mmHg}$ or DBP $>90 \mathrm{mmHg}$

${ }^{\mathrm{i}}$ Estimated based on the Japanese version of the International Physical Activity Questionnaire 
global PSQI score. Among 1346 participants with a global PSQI score $>5.5$ (sleep disturbance group), significantly more participants were women and had a GDS $\geq 6$, severer subjective pain, history of cerebrovascular disease (stroke), weak social support from spouse, family, and friends, a MMSE $<24$, and an eGFR $<60$ compared with those with a global PSQI score $\leq 5.5$ (no-disturbance group). Moreover, alcohol consumption and prevalence of smoking habit were significantly lower in the sleep disturbance group. No significant association was found for medical histories of cancer of any kind, hypertension, and diabetes between the two groups.

In the sex- and 5-year age-adjusted logistic regression model (see Table 3), participants with severe or very severe subjective pain, compared to those with no subjective pain, had the highest OR (3.85, $95 \%$ CI 2.79-5.30) for sleep disturbance. Higher GDS, lower MMSE score, weak social support from spouse, family, or friends, and the presence of a medical history of stroke also showed significant associations, with ORs higher than the null value for sleep disturbance. However, other variables including eGFR $(P=0.07)$, medical histories of AMI $(P=0.09)$, cancer of any kind $(P=0.16)$, diabetes $(P>0.2)$, and hypertension $(P>0.2)$, PA $(P>0.2)$, alcohol consumption $(P>0.2)$, and smoking habit $(P>0.2)$ showed no significant association with sleep disturbance.

Figure 1 illustrates independent variables showing statistically higher ORs after mutual adjustment for sleep disturbance. The variables entered into the multivariate regression model were all independent variables that showed an association with sleep disturbance $(P<0.2)$ (Table 3). Severe or very severe subjective pain, as compared with no pain, showed the highest OR (3.00, $95 \% \mathrm{CI}$ 2.15-4.19), albeit lower than that in the model adjusted for only sex and 5-year age group. Furthermore, ORs of subjective pain increased in a dose-dependent manner, and even the OR of very mild subjective pain showed a significant increase. Participants with a GDS $\geq 6$ and those with GDS 2-5 had a 2.29-fold (95\% CI 1.86-2.81) and 1.36-fold (95\% CI 1.16-1.59) higher OR, respectively, than that of the referent group. Social support from spouse and family, but not from friends, showed an increased OR, which was greater than the null values of 1.21 (95\% CI 1.01-1.44) and 1.44 (95\% CI 1.23-1.70), respectively. The presence of a history of stroke had a statistically significant association with sleep disturbance. Neither the history of myocardial infarction nor cancer of any kind showed an increased OR. Cognitive impairment evaluated by MMSE $<24$ was not a significant independent variable. Renal dysfunction evaluated by eGFR was not statistically significant either. Women showed a 1.56-fold (95\% CI 1.34-1.83) higher OR than men, and only those aged $\geq 80$ years had an increased OR (1.31, $95 \%$ CI 1.01-1.69)
Table 3 Sex and 5-year age-adjusted odds ratios (ORs) with $95 \%$ confidence intervals (CIs) for sleep disturbance (PSQI <5.5) and relationships between sleep disturbance and questionnaire items

\begin{tabular}{|c|c|c|c|c|}
\hline \multirow{2}{*}{$\overline{\text { Sex }}$} & \multirow[t]{2}{*}{ OR } & \multicolumn{2}{|c|}{$95 \% \mathrm{CI}$} & \multirow[t]{2}{*}{$P$ value } \\
\hline & & & & \\
\hline Women/men & 1.61 & 1.41 & 1.84 & 0.00 \\
\hline \multicolumn{5}{|l|}{ Age } \\
\hline $70-74 / 65-69$ & 1.15 & 0.98 & 1.36 & 0.09 \\
\hline $75-79 / 65-69$ & 1.22 & 1.01 & 1.48 & 0.04 \\
\hline$\geq 80 / 65-69$ & 1.56 & 1.23 & 1.96 & 0.00 \\
\hline \multicolumn{5}{|l|}{ Current health condition } \\
\hline \multicolumn{5}{|l|}{ Depression $\left(\mathrm{GDS}^{\mathrm{a}}\right)$} \\
\hline $3-5 / 0-2$ & 1.53 & 1.31 & 1.78 & 0.00 \\
\hline$\geq 6 / 0-2$ & 3.17 & 2.61 & 3.84 & 0.00 \\
\hline \multicolumn{5}{|l|}{ Cognitive impairment $\left(\mathrm{MMSE}^{\mathrm{b}}\right)$} \\
\hline$<24 / \geq 24$ & 1.42 & 1.05 & 1.92 & 0.02 \\
\hline \multicolumn{5}{|l|}{$\mathrm{eGFR}^{\mathrm{c}}$} \\
\hline$\geq 60,<90 / \geq 90$ & 1.06 & 0.81 & 1.41 & 0.67 \\
\hline$<60 / \geq 90$ & 1.32 & 0.98 & 1.79 & 0.07 \\
\hline \multicolumn{5}{|l|}{ Subjective bodily pain ${ }^{\mathrm{d}}$} \\
\hline Very mild/none & 1.37 & 1.12 & 1.67 & 0.00 \\
\hline Mild/none & 1.95 & 1.63 & 2.34 & 0.00 \\
\hline Moderate/none & 2.91 & 2.33 & 3.63 & 0.00 \\
\hline Severe or very severe/none & 3.85 & 2.79 & 5.30 & 0.00 \\
\hline \multicolumn{5}{|l|}{ History of chronic conditions } \\
\hline \multicolumn{5}{|l|}{ Stroke } \\
\hline Yes/no & 1.63 & 1.24 & 2.15 & 0.00 \\
\hline \multicolumn{5}{|l|}{ Myocardial infarction } \\
\hline Yes/no & 1.45 & 0.95 & 2.22 & 0.09 \\
\hline \multicolumn{5}{|l|}{ Diabetes } \\
\hline Yes $/$ no & 1.12 & 0.93 & 1.36 & 0.24 \\
\hline \multicolumn{5}{|l|}{ Hypertension } \\
\hline Yes $/$ no & 0.98 & 0.85 & 1.14 & 0.82 \\
\hline \multicolumn{5}{|l|}{ Cancer of any kind } \\
\hline Yes/no & 1.18 & 0.94 & 1.48 & 0.16 \\
\hline \multicolumn{5}{|l|}{ Social support } \\
\hline \multicolumn{5}{|l|}{ Spouse } \\
\hline Weak/strong & 1.29 & 1.09 & 1.53 & 0.00 \\
\hline \multicolumn{5}{|l|}{ Family } \\
\hline Weak/strong & 1.66 & 1.43 & 1.94 & 0.00 \\
\hline \multicolumn{5}{|l|}{ Friends } \\
\hline Weak/strong & 1.47 & 1.26 & 1.72 & 0.00 \\
\hline \multicolumn{5}{|l|}{ Lifestyle habits } \\
\hline \multicolumn{5}{|l|}{ Physical activity $\left(\right.$ METs $\left.^{\mathrm{g}}\right)$} \\
\hline$\geq 0,<60 / \geq 300$ & 0.97 & 0.80 & 1.18 & 0.78 \\
\hline$\geq 60,<300 / \geq 300$ & 0.90 & 0.72 & 1.12 & 0.34 \\
\hline \multicolumn{5}{|l|}{ Alcohol consumption } \\
\hline$<20 / 0$ & 1.12 & 0.93 & 1.36 & 0.24 \\
\hline $20-40 / 0$ & 0.95 & 0.74 & 1.22 & 0.67 \\
\hline$\geq 40 / 0$ & 0.89 & 0.69 & 1.15 & 0.38 \\
\hline
\end{tabular}


Table 3 continued

\begin{tabular}{lllll}
\hline & OR & $95 \%$ CI & $P$ value \\
\hline Smoking habit & & & & \\
Ex-smoker/current smoker & 1.15 & 0.89 & 1.50 & 0.29 \\
Non-smoker/current smoker & 1.16 & 0.88 & 1.54 & 0.30 \\
\hline
\end{tabular}

${ }^{a}$ Geriatric Depression Scale (GDS-15)

b Mini-mental state examination

${ }^{c}$ Estimated glomerular filtration rate (unit: $\mathrm{ml} / \mathrm{min} / 1.73 \mathrm{~m}^{2}$ )

d Bodily pain included in the MOS 36-item short-form health survey

e Physician-diagnosed diabetes with medical treatment or $\mathrm{HbAlc}$ $>6.5 \%$ at baseline

${ }^{\mathrm{f}}$ Currently treated for SBP $>140 \mathrm{mmHg}$ or DBP $>90 \mathrm{mmHg}$

g Estimated based on the Japanese version of the International Physical Activity Questionnaire

for sleep disturbance when compared with those aged between 65 and 69. No serious multicollinearity was observed (variance inflation factors $<10$ ) in the multivariate model, and the Hosmer-Lemeshow statistic clarified the fit of the data.

\section{Discussion}

In this study, mean global PSQI scores by age groups ranged from 4.37 to 5.04 for male participants, and 5.26 to 5.82 for female participants, which were similar to the results obtained from the general Japanese adult population consisting of the same age groups [21]. Older age groups had higher global PSQI scores, with women scoring higher than men, consistent with previous reports [21, 22]. Furthermore, $36.1 \%$ of our participants $(30.8 \%$ of males and $41.5 \%$ of females) had a global PSQI score $\geq 5.5$ (i.e., definition of sleep disturbance), which is in line with a previous study [37] reporting a total percentage of $37.3 \%$ for male and female community-dwelling elderly individuals aged $\geq 65$ years.

In the present study, we performed multiple logistic regression analysis in which known factors that were reportedly associated with sleep disturbance were mutually adjusted. As a result, in addition to sex and age, bodily pain, depression, spousal support, familial support, and stroke were determined to be independent factors significantly associated with sleep disturbance.

In this study, $68 \%$ of participants complained of bodily pain. Among these, those with the highest self-rated subjective pain (severe or very severe) had a significantly higher OR for sleep disturbance, compared with the nopain group (3.00, $95 \%$ CI 2.15-4.19). People with bodily pain, compared to those without, reportedly have a longer sleep latency, more frequent awakening during sleep, poorer sleep efficiency, and hence, significantly poorer sleep [11]. Consistent with our present study results, one study reported an OR of 1.88-2.68 for insomnia symptoms such as difficulty initiating sleep and difficulty maintaining sleep among those with bodily pain [7]. We did not determine the site or cause of pain in this study. Ten to $88 \%$ of elderly people have chronic pain in their neck, back, joints, legs, or feet [38, 39], and in many cases, the complaint of pain is not confined to a single area, but concerns multiple sites [40]. Osteoarthritis (e.g., knee
Fig. 1 Mutually adjusted odds ratios with $95 \%$ confidence intervals for sleep disturbance using the multiple logistic regression model. Mutually adjusted variables not indicated in this figure include cognitive impairment, eGFR, acute myocardial infarction, cancer of any kind, and social support from friends. Error bars indicate $95 \%$ confidence intervals

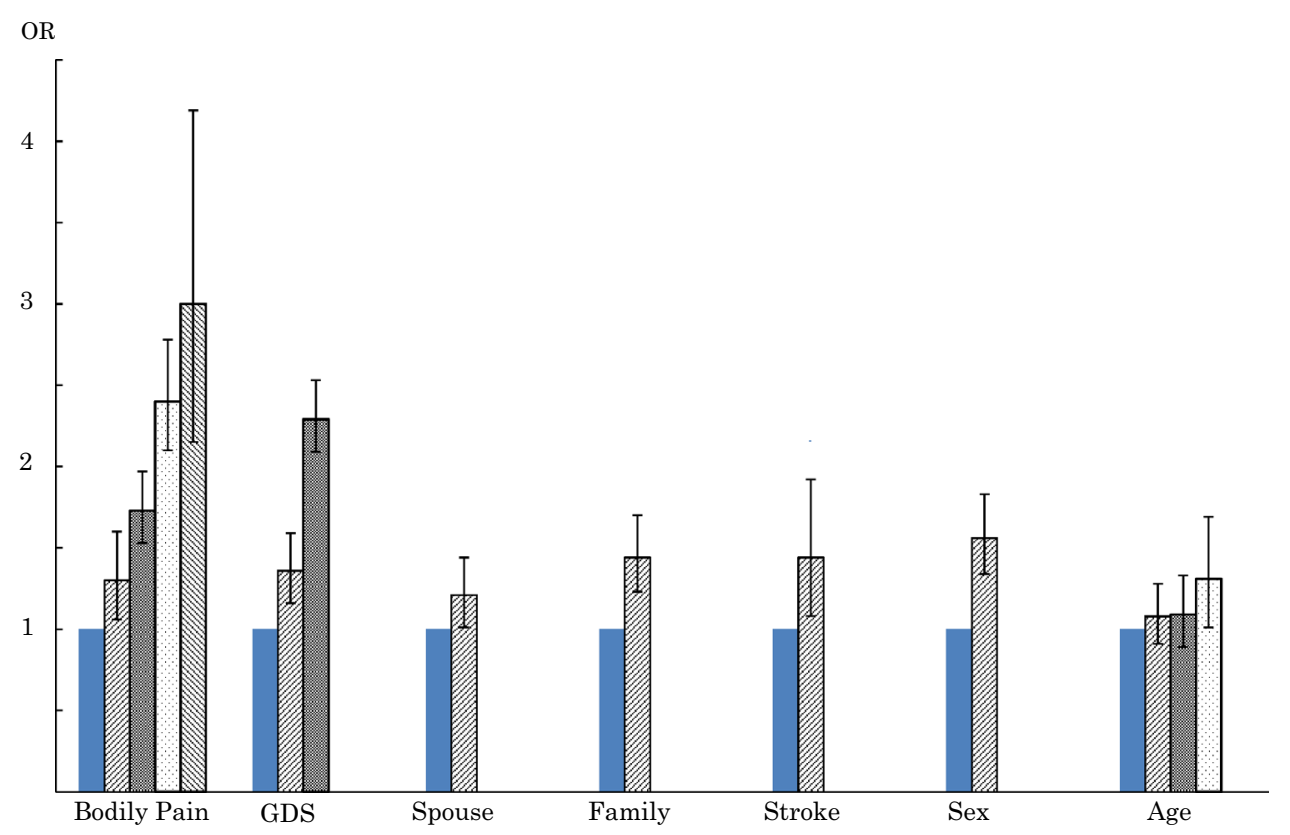


joints), osteoporosis, lumbar spinal canal stenosis, compression fracture, osteoarthritis of the hip, and postherpetic neuralgia are among the assumed causes of pain [39]. In the present study, a higher OR was obtained in groups with increasing severity of subjective bodily pain, which is consistent with a previous study reporting that sleep disturbance increases with increasing frequency of pain and a higher number of pain sites [41]. Notably, even those with very mild bodily pain showed a significantly higher OR (1.30, $95 \%$ CI 1.06-1.60) for sleep disturbance.

Next to bodily pain, the group with a GDS score $\geq 6$ had a significantly higher OR $(2.29,95 \%$ CI $1.86-2.81)$ compared to the group with a GDS score of 0-2. GDS score $\geq 6$ is the cut off value for probable clinical depression among the Japanese elderly [29]. The group with a GDS score 3-5 also showed a significantly higher OR. These results are consistent with a previous study [36], in which a graded association was found between depressive symptoms and sleep disturbance (assessed by the PSQI) with an OR of 2.06 for GDS score 3-5 and 3.68 for GDS score $\geq 6$, as compared with GDS score $0-2$. Since the present study was a cross-sectional study, the temporal relationship between depressive symptoms and sleep disturbance is unclear. A cohort study on elderly subjects [42] reported that individuals with difficulty initiating sleep, compared to those without, developed depression at a significantly higher rate. On the other hand, another cohort study [4] reported that the depressive group developed sleep disturbance significantly more frequently relative to the control group.

We assessed social support using the JMS-SSS, a selfadministered questionnaire developed for Japanese subjects in order to evaluate the association between social support and health in epidemiological studies. This perceived social support scale is based on both structural aspects of social relationships and the availability of functional support [32]. Specifically, responses to 8-10 questions are scored to assess functional support from the spouse or other family members (i.e., social embeddedness), and that from friends (i.e., social network). The JMS-SSS has been used to demonstrate that men with strong support from spouse or family tend to refrain from drinking and smoking [43]. Our results of sex- and age-adjusted analyses showed a significant association between support from friends and sleep disturbance, but after adjusting for other factors in the multivariate analysis, only spousal and familial support remained statistically significant. To the best of our knowledge, this study is the first to obtain these findings regarding sleep disturbance and social support using the questionnaires with confirmed validity, i.e., the PSQI and JMS-SSS. In a study that used an arbitrary questionnaire regarding a "person to consult", those who indicated no one or someone other than their spouse or family had an adjusted OR of 2.3 (95\% CI 1.3-5.0) for self-reported sleep problems, compared to those who indicated their spouse or partner as the person for consultation [44]. Moreover, in old age, a reduction in the size of social networks as well as a decrease in the frequency of contact with network members [16] lead to social isolation, which reportedly affects sleep conditions among the elderly [17]. These results, including our own, indicate that individuals receiving poor support from people closer to them have a higher likelihood of suffering from sleep disturbance. The reasons for this include a significant positive correlation between anxiety symptoms, as assessed by the Geriatric Anxiety Inventory, and global PSQI scores [45]. Individuals with anxiety symptoms tend to wake up during sleep latency, have difficulty maintaining sleep, report early morning awakening [46], and show decreased sleep efficiency [47]. As such, individuals with low support from spouse and family may have difficulty controlling their feelings of anxiety and isolation, which likely causes sleep disturbance.

The history of stroke with clinical symptoms also showed a significant association with sleep disturbance. Post-stroke patients are prone to develop sleep disturbance [48], and may suffer a relapse of sleep disturbance several years after onset of stroke [49]. It is possible that stroke sequelae, such as breathing disorders during sleep [50] and central post-stroke pain that often accompanies strong pain [49], are related to this. It is also likely that sleep disturbance is caused by depressive mood as a reaction to poor physical conditions [51].

Cognitive function decline as assessed by the MMSE showed a significant association with sleep disturbance in sex- and age-adjusted analyses, but not in the multivariate analysis including other factors. The presence/absence of renal dysfunction or diabetes was also not associated with sleep disturbance. Moreover, no association was found with average PA during the past 1 week as assessed by the IPAQ, alcohol consumption, smoking habit, and the history of myocardial infarction, hypertension, or cancer. Contrary to our findings, previous studies have found a significant association with sleep disturbance in those with cognitive function decline [10], renal dysfunction [8], diabetes [5], and moderate exercise [14]. This discrepancy may be due to different factors used for simultaneous adjustment, as well as study methods, subject age, and disease severity.

This study has several limitations. First, due to the crosssectional design, temporal associations could not be determined. Follow-up studies are necessary to verify the present findings. Second, the responses analyzed in the present study were obtained from applicants who likely had a high interest in health. Care should be taken in generalizing the results, although participants of the Fujiwara-kyo study have a similar age distribution to that of the national 
population [20], and our participants were not selected on the basis of sleep disturbance, absence or presence of subjective pain, or other related medical conditions. Third, we did not obtain information on other factors such as cataracts, light exposure profiles, nocturia, restless leg syndrome, medications, or other conditions potentially associated with sleep disturbance [52]. Finally, sleep disturbance was judged based on responses to a self-administered questionnaire. However, we used the PSQI, which is widely used in large-scale epidemiological studies, with confirmed validity regarding its Japanese version [21].

Despite the above limitations, this was a large-scale study with a particular focus on the elderly based on a comprehensive set of measurements, which allowed us to mutually adjust for many factors and independently assess the OR magnitude for each factor. In addition to sex and age, significant factors included bodily pain, social support from spouse or family, depressive symptoms, and history of stroke. Other than age and sex, these factors are all preventable. However, further studies are needed to confirm the time sequence of these factors and sleep disturbance among the elderly.

\section{Compliance with ethical standards}

Conflict of interest All authors declare that they have no conflict of interests.

\section{References}

1. Foley DJ, Monjan AA, Brown SL, Simonsick EM, Wallace RB, Blazer DG. Sleep complaints among elderly persons: an epidemiologic study of three communities. Sleep. 1995;18(6) :425-32.

2. Kim K, Uchiyama M, Okawa M, Liu X, Ogihara R. An epidemiological study of insomnia among the Japanese general population. Sleep. 2000;23(1):41-7.

3. Ohayon MM, Carskadon MA, Guilleminault C, Vitiello MV. Meta-analysis of quantitative sleep parameters from childhood to old age in healthy individuals: developing normative sleep values across the human lifespan. Sleep. 2004;27(7):1255-73.

4. Fok M, Stewart R, Besset A, Ritchie K, Prince M. Incidence and persistence of sleep complaints in a community older population. Int J Geriatr Psychiatry. 2010;25(1):37-45.

5. Nilsson PM, Rööst M, Engström G, Hedblad B, Berglund G. Incidence of diabetes in middle-aged men is related to sleep disturbances. Diabetes Care. 2004;27(10):2464-9.

6. Sepahvand E, Jalali R, Mirzaei M, Kargar Jahromi M. Association between short sleep and body mass index, hypertension among acute coronary syndrome patients in coronary care unit. Glob J Health Sci. 2014;7(3):134-9.

7. Foley D, Ancoli-lsrael S, Britz P, Walsh J. Sleep disturbances and chronic disease in older adults: results of the 2003 national sleep foundation sleep in America survey. J Psychosom Res. 2004;56(5):497-502.

8. Plantinga L, Lee K, Inker LA, Saran R, Yee J, Gillespie B, et al. Association of sleep-related problems with CKD in the United States, 2005-2008. Am J Kidney Dis. 2011;58(4):554-64.
9. Doi Y, Minowa M, Uchiyama M, Okawa M, Kim K, Shibui K, et al. Psychometric assessment of subjective sleep quality using the Japanese version of the Pittsburgh Sleep Quality Index (PSQIJ) in psychiatric disordered and control subjects. Psychiatry Res. 2000;97(2-3):165-72.

10. Yaffe K, Nettiksimmons J, Yesavage J, Byers A. Sleep quality and risk of dementia among older male veterans. Am J Geriatr Psychiatry. 2015;23(6):651-4.

11. Blågestad T, Pallesen S, Lunde LH, Sivertsen B, Nordhus IH, Grønli J. Sleep in older chronic pain patients: a comparative polysomnographic study. Clin J Pain. 2012;28(4):277-83.

12. Kaneita Y, Uchiyama M, Takemura S, Yokoyama E, Miyake T, Harano S, et al. Use of alcohol and hypnotic medication as aids to sleep among the Japanese general population. Sleep Med. 2007;8(7-8):723-32.

13. Asghari A, Kamrava SK, Hemami MR, Jalessi M, Yazdanifard P, Farhadi M, et al. Cigarette smoking habit and subjective quality of sleep. Scimetr. 2015;3(1):e18454.

14. King AC, Pruitt LA, Woo S, Castro CM, Ahn DK, Vitiello MV, et al. Effects of moderate-intensity exercise on polysomnographic and subjective sleep quality in older adults with mild to moderate sleep complaints. J Gerontol A Biol Sci Med Sci. 2008;63(9):997-1004.

15. Kaneita Y, Yokoyama E, Harano S, Tamaki T, Suzuki H, Munezawa T, et al. Associations between sleep disturbance and mental health status: a longitudinal study of Japanese junior high school students. Sleep Med. 2009;10(7):780-6.

16. Cornwell B, Laumann EO, Schumm LP. The social connectedness of older adults: a national profile. Am Sociol Rev. 2008;73(2):185-203.

17. Kent RG, Uchino BN, Cribbet MR, Bowen K, Smith TW. Social relationships and sleep quality. Ann Behav Med. 2015; doi:10. 1007/s12160-015-9711-6.

18. Schubert CR, Cruickshanks KJ, Dalton DS, Klein BEK, Klein R, Nondahl DM. Prevalence of sleep problems and quality of life in an older population. Sleep. 2002;25(8):889-93.

19. Buysse DJ, Reynolds CF III, Monk TH, Berman SR, Kupfer DJ. The Pittsburgh Sleep Quality Index: a new instrument for psychiatric practice and research. Psychiatry Res. 1989;28(2):193-213.

20. Okamoto N, Morikawa M, Okamoto K, Habu N, Hazaki K, Harao $\mathrm{A}$, et al. Tooth loss is associated with mild memory impairment in the elderly: the Fujiwara-kyo study. Brain Res. 2010;1349:68-75.

21. Doi Y, Minowa M, Uchiyama M, Okawa M. Subjective sleep quality and sleep problems in the general Japanese adult population. Psychiatry Clin Neurosci. 2001;55(3):213-5.

22. Asghari A, Farhadi M, Kamrava SK, Ghalehbaghi B, Nojomi M. Subjective sleep quality in urban population. Arch Iran Med. 2012;15(2):95-8.

23. Doi Y, Minowa M, Uchiyama M, Okawa M. Development of the Japanese version of the Pittsburgh Sleep Quality Index (PSQI-J). Jpn J Psychiatr Treat. 1998;13(6):755-63.

24. Backhaus J, Junghanns K, Broocks A, Riemann D, Hohagen F. Test-retest reliability and validity of the Pittsburgh Sleep Quality Index in primary insomnia. J Psychosom Res. 2002;53(3):737-40.

25. Ware JE Jr, Sherbourne CD. The MOS 36-item short-form health survey (SF-36) I. Conceptual framework and item selection. Med Care. 1992;30(6):473-83.

26. Huskisson EC. Measurement of pain. Lancet. 1974;304(7889):1127-31.

27. Fukuhara S, Ware JE Jr, Kosinski M, Wada S, Gandek B. Psychometric and clinical tests of validity of the Japanese SF-36 health survey. J Clin Epidemiol. 1998;51(11):1045-53.

28. Sheikh JI, Yesavage JA. Geriatric Depression Scale (GDS); recent evidence and development of a shorter version. Clin Gerontol. 1986;5(1):165-73.

29. Niino N. A Japanese translation of the geriatric depression scale. Clin Gerontol. 1991;10(3):85-7. 
30. Folstein MF, Folstein SE, McHugh PR. "Mini-mental state" a practical method for grading the cognitive state of patients for the clinician. J Psychiatr Res. 1975;12(3):189-98.

31. Maki N, Ikeda M, Hokoishi K, Nebu A, Komori K, Hirono N, et al. The validity of MMSE and SMQ as screening tests for dementia in the elderly general population-a study of one rural community in Japan. Dement Geriatr Cogn Disord. 2000;11(4):193-6.

32. Tsutsumi A, Kayaba K, Ishikawa S, Kario K, Matsuo H, Takuma S. Jichi Medical School Social Support Scale (JMS-SSS) revision and tests for validity and reliability. Jpn J Public Health. 2000;47(10):866-78.

33. Guidelines for Data Processing and Analysis of the International Physical Activity Questionnaire (IPAQ)-Short Form. Version 2.0. 2004. http://www.ipaq.ki.se/.

34. Kouda K, Iki M, Fujita Y, Tamaki J, Yura A, Kadowaki E, et al. Alcohol intake and bone status in elderly Japanese men: baseline data from the Fujiwara-kyo osteoporosis risk in men (FORMEN) study. Boon. 2011;49(2):275-80.

35. Clinical practice guidebook for diagnosis and treatment chronic kidney disease. Nihon Jinzo Gakkai Shi. 2012;54:1034-191 (in japanese).

36. Paudel ML, Taylor BC, Diem SJ, Stone KL, Ancoli-Israel S, Redline $\mathrm{S}$, et al. Association between depressive symptoms and sleep disturbances in community-dwelling older man. J Am Geriatr Soc. 2008;56(7):1228-35.

37. Sukegawa $\mathrm{T}$, Itoga $\mathrm{M}$, Seno $\mathrm{H}$, Miura S, Inagaki T, Saito W, et al. Sleep disturbances and depression in the elderly in Japan. Psychiatry Clin Neurosci. 2003;57(3):265-70.

38. Nakamura M, Nishiwaki Y, Ushida T, Toyama Y. Prevalence and characteristics of chronic musculoskeletal pain in Japan. J Orthop Sci. 2011;16(4):424-32.

39. Helme RD, Gibson SJ. The epidemiology of pain in elderly people. Clin Geriatr Med. 2001;17(3):417-31.

40. Herr KA, Mobily PR, Wallace RB, Chung Y. Leg pain in the rural Iowa $65+$ population. Prevalence, related factors, and association with functional status. Clin J Pain. 1991;7(2):114-21.
41. Zarit SH, Griffiths PC, Berg S. Pain perceptions of the oldest old: a longitudinal study. Gerontologist. 2004;44(4):459-68.

42. Yokoyama E, Kaneita Y, Saito Y, Uchiyama M, Matsuzaki Y, Tamaki $\mathrm{T}$, et al. Association between depression and insomnia subtypes: a longitudinal study on the elderly in Japan. Sleep. 2010;33(12):1693-702.

43. Tsutsumi A, Tsutsumi K, Kayaba K, Igarashi M. Health-related behaviors, social support, and community morale. Int J Behav Med. 1998;5(2):166-82.

44. Nomura K, Yamaoka K, Nakao M, Yano E. Social determinants of self-reported sleep problems in South Korea and Taiwan. J Psychosom Res. 2010;69(5):435-40.

45. Yu J, Rawtaer I, Fam J, Jiang MJ, Feng L, Kua EH, et al. Sleep correlates of depression and anxiety in an elderly Asian population. Psychogeriatrics. 2015;. doi:10.1111/psyg.12138.

46. Leblanc MF, Desjardins S, Desgagne A. Sleep problems in anxious and depressive older adults. Psychol Res Behav Manag. 2015;8:161-9.

47. Spira AP, Stone K, Beaudreau SA, Ancoli-Israel S, Yaffe K. Anxiety symptoms and objectively measured sleep quality in older women. Am J Geriatr Psychiatry. 2009;17(2):136-43.

48. Palomäki H, Berg A, Meririnne E, Kaste M, Lönnqvist R, Lehtihalmes M, et al. Complaints of poststroke insomnia and its treatment with mianserin. Cerebrovasc Dis. 2003;15(1-2):56-62.

49. Jönsson AC, Lindgren I, Hallström B, Norrving B, Lindgren A. Prevalence and intensity of pan after stroke: a population based study focusing on patients' perspectives. J Neurol Neurosurg Psychiatry. 2006;77(5):590-5.

50. Mohsenin V. Sleep-related breathing disorders and risk of stroke. Stroke. 2001;32(6):1271-8.

51. Salter K, Mehta S, Bhogal S, Teasell R, Foley N, Speechley M. Post stroke depression. Expert Rev Neurother. 2008;8:75-92.

52. Kamel NS, Gammack JK. Insomnia in the elderly: cause, approach, and treatment. Am J Med. 2006;119:463-9. 